चि INDEX

\section{PAR AUTEURS}

A

\begin{abstract}
Abina M.-A. Voir Fernandez N.
Adoutte A. Voir Philippe $\mathrm{H}$.

Agut H. Voir Dupin N.

Algarté M. Voir Costello R.

Allamand V. Voir Richard I.

Allamand V. Voir Duclos F.

Alloisio N. Voir Delaunay J.

Amiel J. Voir Attié T.
\end{abstract}

Amigorena S. Transport intracellulaire des molécules de classe $\|$ du CMH. 11 $\left(n^{\circ} 5\right): 661-8$-as.

Amiranoff B. Insectes, vers, protéases et apoptose. $11\left(n^{\circ} 12\right)$ : 1757-nouv.

Amouyel $\mathbf{P}$. Les allèles du gène de l'apolipoprotéine $E$ et la maladie de CreutzfeldtJakob. $11\left(n^{\circ} 3\right)$ : 484-6-nouv.

Aoudjit F. Rôle du CD40 dans l'activation des lymphocytes B. $11\left(n^{\circ} 2\right)$ : 292-3-nouv.

Artigas F. Voir Gardier A.M.

Arveiler B. Voir Lacombe D.

Attié T. Endothélines et leurs récepteurs :

de nouveaux acteurs du développement.

$11\left(n^{\circ} 8\right)$ : 1172-5-nouv

Auberger P. Voir Mari B.

Aubin J.-T. Voir Dupin N.

Aunis D. Voir Vitale N.

Auroux M. Environnement, spermatozoï-

de et descendance. $11\left(n^{\circ} 4\right)$ : 571-7-as.

Auxillien S. Édition des ARN d'euca-

ryotes et viraux par désamination enzyma-

\section{ABRÉVIATIONS}

as : article de synthèse

$b r:$ brève

$c:$ courrier

$c g:$ chronique génomique

$d t$ : dossier technique

$e d$ : éditorial

$f c:$ faits et chiffres

$h d$ : hypothèse/débat

$h m s$ : histoire de la médecine et des sciences

hom : hommage

lo: libre opinion

$l t:$ lettre à $\mathrm{m} / \mathrm{s}$

mns : mini-synthèse

no: note originale

nouv: nouvelle

$p n$ : prix Nobel

$s f g$ : société française de génétique

$u b g:$ ultra-brève génétique tique d'adénosines en inosines. $11\left(n^{\circ} 8\right)$ : 1089-98-as.

Avallet O. Voir Saez J.M.

\section{B}

Babinet C. Voir Colucci-Guyon E.

Babinet C. Un nouveau pas dans I'utilisation du système Cre-LoxP chez les cellules souches embryonnaires de souris : la création de remaniements chromosomiques. $11\left(n^{\circ} 8\right)$ : 1154-7-mns.

Bach J.-F. Voir Chatenoud L.

Bader M.-F. Voir Vitale N.

Bahraoui E. Voir Moulard M.

Baptist M. Voir Maenhaut C.

Barbaux S. Le point sur le déterminisme du sexe chez les mammifères. $11\left(n^{\circ} 4\right)$ : 529-36-as.

Beaudet A. Voir Descarries L.

Beckmann J.S. Voir Duclos F.

Beckmann J.S. Voir Richard I.

Becq F. Voir Merten M.

Bédard M.-A. Sciences cognitives et neu ropharmacologie. $11\left(n^{\circ} 11\right)$ : 1515-7-ed.

Bédard M.-A. Neurochimie des troubles cognitifs dans la maladie de Parkinson. 11 (n० 11) : 1541-9-as.

Benarous R. L'interaction de la protéine Nef-VIH avec la protéine cellulaire $\beta$-COP : un détournement de la machinerie du trafic intracellulaire et d'exocytose au profit du VIH ? $11\left(n^{\circ} 3\right)$ : 478-9-nouv.

Benichou B. Voir Bürglen L.

Benichou S. Voir Benarous R.

Bensussan A. Voir Hérold C.

Berberof $M$. Voir Pays $E$.

Bergeron M. Médecine, science et génétique: les années charnières. $11\left(n^{\circ} 1\right): 10$ 1-ed.

Bergeron M. médecine/sciences a 10 ans la médecine et la science dans dix ans. 11 $\left(n^{\circ} 3\right)$ : 315-6-ed.

Bergeron M. Claude Vézina, 1926-1995 11 ( $\left.n^{\circ} 11\right)$ : 1629-hom.

Bergmann É. Voir Roy R.

Bernard J. France Quéré, 1936-1995. 11 (n०5): 793-hom.

Berta $\mathbf{P}$. Les gènes $S O X$ : retour vers la détermination du sexe. $11\left(n^{\circ} 2\right): 300$ nouv.

Berthelot P. Voir Kerleau M.

Berthoux L. Voir Darlix J.-L.

Bertrand-Servais M. Voir Letur-Könirsch H.

Blanc G. Voir Tassin J.-P.

Bockaert J. Les récepteurs à sept do maines transmembranaires: physiologie et pathologie de la transduction. $11\left(n^{\circ} 3\right)$ 382-94-as.

Boeynaems J.-M. Un rôle physiologique des plasmalogènes : la protection contre le stress oxydatif et l'excès d'iode. 11 $\left(n^{\circ} 2\right)$ : 254-9-as.

Boisacq-Schepens $\mathbf{N}$. La faculté de médecine de l'université catholique de Louvain. $11\left(n^{\circ} 9\right)$ : 1219-23-ed.

Boitier E. Voir Oudard S.

Bol A. Voir Vanoverschelde J.-L.

Bonnaventure J. Voir Dreyfus J.-C.

Bonne G. Vers une meilleure compréhension du rôle de la protéine $C$ dans la contraction cardiaque. $11\left(n^{\circ} 12\right): 1739-41$ nouv.

Bonneau D. Voir Kaplan J.

Boon $\mathbf{T}$. Antigènes de rejet des tumeurs et immunothérapie spécifique du cancer. 11 ( $\left.n^{\circ} 9\right)$ : 1279-87-as.

Borgeat P. L'adénosine, un anti-inflammatoire naturel. $11\left(n^{\circ} 2\right)$ : 294-nouv.

Borgers M. Voir Vanoverschelde J.-L.

Bouchard P. Voir Christin-Maître S.

Boumsell L. Voir Hérold C.

Bourg N. Voir Richard I.

Bourg N. Voir Duclos F.

Bourgoin S.-G. Régulation de la phospholipase $D$ par les protéines $G$ de faible poids moléculaire : réalités et perspectives. 11 ( $\left.n^{\circ} 3\right)$ : 476-7-nouv.

Bouvier $\mathbf{M}$. Le récepteur $\beta_{2}$-adrénergique: un modèle d'étude des mécanismes moléculaires de la désensibilisation. $11\left(n^{\circ} 6\right)$ : 819-27-as.

Braekman J.-C. Voir Boeynaems J.-M.

Bréchot C. Virus des hépatites: la saga d'une famille nombreuse au $x x^{\mathrm{e}}$ siècle. 11 (n० 10) : 1373-7-ed.

Brenguier L. Voir Richard I.

Briard M.-L. Voir Kaplan J.

Brichard V.G. Voir Boon T.

Brouillet E. La neurotoxicité du 3-nitro propionate chez le primate confirme l'hy pothèse d'un mécanisme excitotoxique indirect dans l'étiologie de la maladie de Huntington. 11 ( $\left.n^{\circ} 10\right)$ : 1489-91-nouv.

Broux O. Voir Richard I.

Broux O. Voir Duclos F.

Bruyns C. Voir Gérard C.

Bürglen L. Identification et caractérisation d'un gène déterminant dans les amyotrophies spinales. $11\left(n^{\circ} 1\right): 149-51$-nouv.

Burlet P. Voir Bürglen L

Bursaux E. Boire un petit coup, c'est doux ! $11\left(n^{\circ} 1\right): 124-5-f c$.

Bursaux E. A quoi servent les œstrogènes chez l'homme ? $11\left(n^{\circ} 1\right): 126-7$ nouv.

Bursaux E. Transition démographique en Extrême-Orient. 11 ( $n^{\circ} 3$ ) : 465-6-fc.

Bursaux E. Pleins feux sur la synthèse des cytokines inflammatoires. $11\left(n^{\circ} 3\right)$. 467-9-nouv.

Bursaux E. L'homme et l'eau. $11\left(n^{\circ} 5\right)$ : 761-3-fc.

Bursaux E. Le National Cancer Institute (NCI) sur la sellette. $11\left(n^{\circ} 6\right):$ 901-fc.

Bursaux E. Inégalités familiales et inégalités sociales de santé. $11\left(n^{\circ} 7\right)$ : 1037-8-fc. Bursaux E. Le syndrome de Turcot à I'heure des gènes de prédisposition aux cancers. $11\left(n^{\circ} 7\right)$ : 1044-nouv.

Bursaux E. Une expérience américaine de contrôle des dépenses de médicaments: effet de l'autorisation sur l'usage des antiinflammatoires non stéroïdiens. $11\left(n^{\circ} 8\right)$ : 1165-6-fc.

Bursaux E. Mutation du gène de la carboxypeptidase $\mathrm{E}$ et obésité. $11\left(n^{\circ} 8\right)$ : 1171-nouv.

Bursaux E. Voir Mignon A

Bursaux E. Virus d'Epstein-Barr et carcinome naso-pharyngé. Rôle de LMP-1. 11 $\left(n^{\circ} 12\right)$ : 1765-6-nouv.

Bursaux E. Inégalités des femmes. 11 $\left(n^{\circ} 1\right): 1594-5-f c$. 
Butor C. Immunité locale et vaccination. $11\left(n^{\circ} 5\right)$ : 703-11-as.

Cadore B. Questions éthiques à propos des greffes expérimentales de tissus fœtaux sur les souris scid/scid. $11\left(n^{\circ} 5\right)$ : 75560-10.

Caen J.-P. Les glycoprotéines plaquettaires : vingt ans après. $11\left(n^{\circ} 7\right)$ : 1021-6mns.

Cahn J. Henri Laborit nous a quittés. 11 $\left(n^{\circ} 7\right)$ : 1056-c.

Cahour A. Les perspectives d'un vaccin contre l'hépatite $C$ : quelle stratégie adopter? $11\left(n^{\circ} 1\right): 81-91$-as.

Caira F. L'enzyme bifonctionnelle hydra tase/déshydrogénase et l'oxydation peroxysomiale des acides gras à très longue chaîne. 11 ( $\left.n^{\circ} 8\right)$ : 1131-9-as.

Calvez V. Voir Dupin N.

Campbell K.P. Voir Duclos F.

Camuzat A. Voir Kaplan J.

Capiod T. Des portes s'ouvrent sur les allées et venues des ions calcium. $11\left(n^{\circ} 7\right)$ 1027-33-mns.

Capron A. Le langage moléculaire des parasites. $11\left(n^{\circ} 3\right): 431$-9-as.

Carbon P. Voir Sturchler-Pierrat C

Carbonne B. Voir Sornette D.

Carillo S. Le catabolisme protéique intracellulaire: une fonction biologique majeu re. Partie 1: Les mécanismes de dégradation. $11\left(n^{\circ} 5\right)$ : 723-34-as.

Carillo S. La catabolisme protéique intracellulaire : une fonction biologique majeu re. Partie 2: exemples de dégradation conditionnelle et genèse des peptides antigéniques. 11 ( $\left.n^{\circ} 6\right)$ : 845-52-as.

Carosella E Voir Gluckman E.

Carrier L. La cardiomyopathie hypertro phique familiale: maladie du sarcomère cardiaque? $11\left(n^{\circ} 12\right): 1685-93$-as.

Carrier L. Voir Bonne G.

Cassiers L. Voir Boisacq-Schepens N.

Cavadore J.-C. Voir Darbon J.-M.

Cesbron J.-Y. Voir Cadore B.

Chabannon C. Les cellules souches hématopoḯtiques du sang périphérique chez l'homme. $11\left(n^{\circ} 1\right): 17-27-a s$.

Chabre 0. Une mutation de la protéine Gs $\alpha$ responsable d'hyper- et d'hypofonctionnements endrocrines. $11\left(n^{\circ} 1\right): 105-8$ mns.

Chambon C. Production d'érythropoïétine humaine: de nouvelles voies d'administration de cette hormone par thérapie génique. $11\left(n^{\circ} 3\right)$ : 495-6-nouv.

Charnay P. Voir Topiko P.

Chatenoud $\mathbf{L}$. Peut-on rétablir la tolérance au soi dans les maladies auto-immunes? $11\left(n^{\circ} 11\right)$ : 1557-65-as.

Chauveau D. Voir Richard S

Cherkaoui Malki M. Voir Caira F.

Chiannikulchai N. Voir Richard I.

Chomel J.-C. Mécanismes de l'action on-

cogénique de Bcr-Abl dans la leucémie myéloïde chronique. $11\left(n^{\circ} 12\right): 1669-78$ as.

Christin-Maître S. La contraception masculine. $11\left(n^{\circ} 4\right)$ : 579-90-as.
Clermont O. Voir Bürglen L.

Closson V. Voir Zalcman G.

Cohen D. Voir Bürglen L.

Cohen D. Voir Richard I.

Collenot A. Voir Denis $\mathrm{H}$.

Colucci-Guyon E. A la recherche d'une fonction pour les filaments intermédiaires. $11\left(n^{\circ} 8\right)$ : 1144-9-mns.

Communi D. Voir Erneux C.

Concordet J.-P. Développement des vertébrés : pas de notochorde en l'absence du facteur de transcription HNF3b. 11 $\left(n^{\circ} 1\right)$ : 134-5-nouv.

Conquet F. Glutamate et mGluR1 : la clé et la serrure de l'apprentissage chez les mammifères. $11\left(n^{\circ} 2\right)$ : 287-90-nouv.

Coppin C. L'ostéogenèse imparfaite : des mutations aux phénotypes. $11\left(n^{\circ} 6\right): 853$ 9-as.

Corabœuf E. Voir Mercadier J.-J.

Cornélis G.R. Le plasmide pYV, élément clé de la virulence des Yersinia. $11\left(n^{\circ} 9\right)$ 1295-304-as.

Cornillon B. Voir Eldin P

Costagliola J. Réponse à l'essai d'analyse épistémologique de la théorie synthétique de l'évolution. $11\left(n^{\circ} 4\right): 643-c$

Costello R. Activation du système de facteurs de transcription Rel/NF- $\kappa$ B. $11\left(n^{\circ} 7\right)$ 957-65-as.

Couëdel-Courteille A. Voir Butor C

Coulombel L. Les cellules souches hématopoiétiques. $11\left(n^{\circ} 1\right): 13-6$-ed.

Courseaux A. De l'ADN aux gènes: un éventail de techniques. $11\left(n^{\circ} 12\right): 1707$ $16-d t$.

Courtoy P.J. Voir Marbaix E.

Courtoy P.J. Voir Cupers P.

Cozzani E. Voir Nicolas J.-F.

Crépel F. Le monoxyde d'azote. $11\left(n^{\circ} 12\right)$ : 1639-42-ed.

Crommelinck M. Voir Roucoux A

Cruaud C. Voir Bürglen L.

Cupers P. La clathrine est-elle superflue pour l'endocytose non spécifique? 11 $\left(n^{\circ} 9\right)$ : 1271-8-as.

Cuzin F. Voir Sage J.

Dambly-Chaudière C. Voir Ghysen A

Dandolo L. Empreinte parentale des gènes $H 19$ et Igf2. $11\left(n^{\circ} 10\right)$ : 1483-6-nouv Darbon J.-M. De nouveaux régulateurs du cycle cellulaire: les protéines modu latrices des complexes cdk-cyclines. 11 (n'3) : 349-56-as.

Darlix J.-L. La nucléocapside du VIH-1: un paradigme pour la recherche et ses applications médicales. $11\left(n^{\circ} 3\right): 420-9$-as.

De Lamirande E. Dérivés actifs de l'oxy gène et fonctions du spermatozoïde. 11 $\left(n^{\circ} 4\right)$ : 564-9-as.

De Smedt F. Voir Erneux

De Verneuil H. Les porphyries héréditaires : de la pathologie moléculaire à la thérapie génique. $11\left(n^{\circ} 6\right): 873-8-m n s$.

Debatisse $M$. Amplification génique, plasticité des génomes et oncogenèse. 11 (n०8) : 1099-109-as.

Debré P. Voir Paul-Eugène Dugas N.

Degos L. Un pionnier de la médecine scientifique, Alfred Donne. $11\left(n^{\circ} 10\right)$ : 1478-81-hms.

Delaunay $J$. L'allèle $a^{L E L Y}$, un allèle tranquille, mais dangereux, du gène $\alpha$-spectrine érythroïde. $11\left(n^{\circ} 5\right): 752-4-m n s$

Deliège D. Médecins et médecines: crois sance et enjeux politiques pour l'avenir. 11 $\left(n^{\circ} 9\right)$ : 1333-8-fc.

Delamur E. Un être vivant dont le génome vient d'être entièrement séquencé: Haemophilus influenzae Rd. 11 ( $\left.n^{\circ} 12\right)$ : 1761-nouv.

Denamur E. Les gènes des chimpanzés nous livrent les secrets de leur vie. 11 $\left(n^{\circ} 2\right)$ : 298-nouv.

Denamur E. Le mystère de l'homogénéité génétique de l'humanité. $11\left(n^{\circ} 5\right): 770-1$ nouv.

Denamur E. Infections bactériennes chez la souris mucoviscidosique : un modèle de la maladie pulmonaire humaine. $11\left(n^{\circ} 7\right)$. 1046-7-nouv.

Denamur E. De la génétique des populations bactériennes à l'épidémiologie des maladies infectieuses. $11\left(n^{\circ} 10\right): 1399$ 406-as.

Denis F. Découverte de nouveaux virus des hépatites, les GBV : quelle est leur pla ce et quel est leur pouvoir pathogène ? 11 $\left(n^{\circ} 6\right)$ : 883-5-mns

Denis H. Taxinomie et embryologie: une connexion ancienne et fructueuse. 11 (n०12): 1695-706-hd.

Denis H. La théorie des feuillets : implications embryologiques et évolutives. 11 (n०11): 1581-93-hd.

Denis M.G. Polypose adénomateuse familiale et gène $A P C .11\left(n^{\circ} 3\right)$ : 443-6-mns. Depré C. Voir Vanoverschelde J.-L.

Descarries L. Les neurones à monoamine du cerveau. $11\left(n^{\circ} 6\right): 817-8-e d$.

Desnoyers S. Voir Girish S.

Deutsch J. A la recherche de la fonction de I'homologue mammifère du gène de drosophile Suppressor of Hairless. 11 (n०3) : 441-2-mns.

Deutsch J. Le fond de l'œil: l'œil de la drosophile est-il homologue de celui de la souris? $11\left(n^{\circ} 10\right)$ : 1447-52-mns.

Deutsch J. La reconnaissance de la génétique du développement. $11\left(n^{\circ} 11\right): 1625$ 8-pn.

Devaud C. Voir Richard I.

Deybach J.-C. Voir de Verneuil H.

Dion R. Voir Vanoverschelde J.-L.

Dollfus H. Voir Kaplan J.

Donnez J. Voir Marbaix E.

Donzé O. Voir Gingras A.-C.

Dorseuil 0 . Un rôle physiologique pour Bcr. $11\left(n^{\circ} 7\right)$ : 1039-40-nouv.

Dreyfus J.-C. Les lésions moléculaires des types 1 et 2 du syndrome de Waardenburg. $11\left(n^{\circ} 1\right)$ : 133-nouv.

Dreyfus J.-C. Acide rétinoïque et vieillissement cutané. $11\left(n^{\circ} 1\right)$ : 140-1-nouv.

Dreyfus J.-C. Taille des télomères et génétique. $11\left(n^{\circ} 2\right)$ : 279-81-nouv.

Dreyfus J.-C. Les dystrophies maculaires de la rétine. $11\left(n^{\circ} 2\right): 295-n o u v$.

Dreyfus J.-C. Des dangers du déficit en cholestérol. $11\left(n^{\circ} 3\right)$ : 470-1-nouv.

Dreyfus J.-C. Enfin un modèle animal apparemment pertinent de maladie d'Alzheimer! $11\left(n^{\circ} 4\right)$ : 627-8-nouv. 
Dreyfus J.-C. Jacques Kruh, 1922-1995. $11\left(n^{\circ} 4\right)$ : 641-hom.

Dreyfus J.-C. Transglutaminase 1 et ichthyose lamellaire. $11\left(n^{\circ} 5\right)$ : 765-6-nouv.

Dreyfus J.-C. Pseudohermaphrodisme masculin dû à une anomalie du récepteur de l'hormone lutéinisante LH. $11\left(n^{\circ} 5\right)$ 774-5-nouv.

Dreyfus J.-C. Des anomalies du récepteur 3 des facteurs de croissance fibroblas tique sont responsables d'une chondrodysplasie létale: le nanisme thanatophore. $11\left(n^{\circ} 5\right)$ : 780-1-nouv.

Dreyfus J.-C. Un gène imprimé dans le diabète? $11\left(n^{\circ} 5\right)$ : 782-4-nouv.

Dreyfus J.-C. Un locus autosomique prédisposant aux délétions de l'ADN mitochondrial. $11\left(n^{\circ} 5\right)$ : 785-6-nouv.

Dreyfus J.-C. Traitement chélateur ora de la surcharge en fer. $11\left(n^{\circ} 6\right)$ : 909-nouv. Dreyfus J.-C. Ultra-brèves-génétique. 11 $\left(n^{\circ} 6\right)$ : 925-ubg.

Drüeke T.B. Anomalies de la croissance du tissu parathyroïdien dans l'hyperparathyroïdie primaire et secondaire. $11\left(n^{\circ} 7\right)$. 1041-nouv.

Duclos F. $\beta$-sarcoglycane: une protéine du complexe dystrophine-glycoprotéines est responsable d'une forme récessive de dystrophie musculaire. $11\left(n^{\circ} 12\right): 1732-8$ mns.

Ducot B. Voir Spira A.

Duffour M.-T. Voir Fernandez N.

Dufier J.-L. Voir Kaplan J.

Dugas B. Voir Paul-Eugène/Dugas N.

Dulioust E. Voir Auroux M.

Dumont J.E. La recherche biomédicale à I'Université Libre de Bruxelles. $11\left(n^{\circ} 2\right)$ 164-8-ed.

Dumont J.E. Voir Maenhaut C

Dupaix A. Voir Jégou B.

Dupin $\mathbf{N}$. Détection de séquences d'ADN apparentées aux virus herpès au cours de la maladie de Kaposi. $11\left(n^{\circ} 6\right)$ : 914-6 nouv.

Durand B. Un nouveau transactivateu des gènes du $\mathrm{CMH}-\|$ muté dans une forme d'immunodéficience héréditaire. $11\left(n^{\circ} 9\right)$ : 1344-5-nouv.

Durand P. Voir Saez J.M.

Durocher D. Rétinoïdes et bases moléculaires des malformations cardiaques congénitales. $11\left(n^{\circ} 1\right)$ : 132-nouv.

Durocher D. Rôle anti-arythmogène de I'endothéline dans l'ischémie cardiaque. $11\left(n^{\circ} 2\right)$ : 291-nouv.

Durocher D. Voir Grépin C.

\section{E}

Earnschaw W. Voir Girish S. Éclache V. Voir Raphaël M. Édery P. Voir Attié T.

Eeckhout Y. Voir Coppin C.

Eeckhout $Y$. Voir Marbaix E

Egly J.-M. Voir Roy R.

Elbaz A. Voir Fontaine B.

Eldin P. Une nouvelle jeunesse pour les myosines. $11\left(n^{\circ} 7\right)$ : 1005-16-as.

Elhabazi A. Voir Hérold C.

Eliaou J.-F. Voir Pinet V.

Émilie D. Nouvelles perspectives des re- cherches sur la polyarthrite rhumatoïde. $11\left(n^{\circ} 11\right)$ : 1577-80-mns.

Erneux C. Implication des phosphatidy inositols et de leurs produits d'hydrolyse dans la signalisation cellulaire. $11\left(n^{\circ} 2\right)$ 240-6-as.

Errera M. Voir Dumont J.E.

Escande J.-P. Voir Dupin N.

\section{$\mathbf{F}$}

Fanchin R. Voir Letur-Könirsch $\mathrm{H}$.

Fardeau M. Voir Richard I.

Fardeau M. Voir Fontaine B.

Fardeau M. Dystrophies musculaires congénitales: une percée. $11\left(n^{\circ} 12\right): 1679$ 84-as.

Fardeau M. Voir Duclos F.

Fardeau-Gauthier M. Voir Kerleau M.

Fellous M. Voir Barbaux S.

Fernandez N. Vaccination antitumorale et thérapie génique. $11\left(n^{\circ} 7\right): 975-83$-as.

Ferré F. Voir Sornette D.

Ferveur J.-F. Attraction sexuelle et structures cérébrales chez les mâles Drosophila melanogaster. 11 ( $n^{\circ} 5$ ) : 767-9-nouv.

Fesquet D. Voir Darbon J.-M.

Feuillard J. Voir Raphaël M.

Fischer A. Voir Hivroz C.

Flis-Treves $\mathbf{M}$. Voir Letur-Könirsch $\mathrm{H}$.

Flori Y.-A. Voir Kerleau M.

Follézou J.-Y. Voir Paul-Eugène/Dugas N.

Fontaine B. Paralysie périodique hypokaliémique et récepteur des dihydropyridines : un rôle inattendu pour un canal calcium. $11\left(n^{\circ} 7\right)$ : 967-73-as.

Fontès M. Voir Courseaux A

Forget H. Voir Lupien S.

Fougerousse F. Voir Richard I.

Foulkes N.S. Voir Masquilier D.

Frézal J. Voir Bürglen L.

Frézal J. Voir Kaplan J.

Froguel $\mathbf{P}$. Une mutation du gène du récepteur du glucagon dans le diabète non insulinodépendant. $11\left(n^{\circ} 3\right)$ : 488-9-nouv.

Frydman R. Voir Letur-Könirsch $\mathrm{H}$.

\section{G}

Gacon G. Les protéines Rac : oncoprotéines et régulateurs du cytosquelette d'actine. 11 ( $\left.n^{\circ} 7\right)$ : 1045-nouv.

Gagnon C. Voir de Lamirande E.

Galanaud P. Voir Richard Y.

Gardier A.M. Base neurobiologique du rôle des récepteurs $5-\mathrm{HT}_{1 \mathrm{~A}}$ dans le mode d'action des antidépresseurs sérotoninergiques. $11\left(n^{\circ} 10\right)$ : 1407-17-as.

Gaussin V. Voir Hue L.

Ged C. Voir de Verneuil H.

Geneviève Lemaire. Voir Crépel F.

Gérard C. Interleukine 10 : facteur favorisant l'échappement d'une tumeur à la surveillance immunitaire? ou facteur favorisant son rejet? $11\left(n^{\circ} 10\right): 1431-5$-as.

Gerber B. Voir Vanoverschelde J.-L.

Gerber S. Voir Kaplan J.

Germot A. Voir Philippe H.

Ghohestani R. Voir Nicolas J.-F.

Ghysen A. Le développement des or- ganes sensoriels chez la drosophile. 11 $\left(n^{\circ} 2\right)$ : 178-88-as.

Gilgenkrantz $H$. Les mutants déficients en rapsyne révèlent un rôle essentiel inattendu de cette protéine. $11\left(n^{\circ} 12\right): 1741-2$ nouv.

Gilgenkrantz $\boldsymbol{H}$. Un nouveau partenaire de la dystrophine musculaire: la NO synthase. $11\left(n^{\circ} 12\right)$ : 1745-nouv.

Gilgenkrantz $H$. Deux promoteurs supplémentaires dans le gène de la dystrophine! 11 ( $\left.n^{\circ} 8\right)$ : 1169-70-nouv.

Gilgenkrantz H. L'injection intrathy mique néonatale d'adénovirus recombi nant: une voie de tolérisation. $11\left(n^{\circ} 10\right)$ 1371-2-It.

Gilgenkrantz S. La génétique, science du futur, se penche sur le passé. $11\left(n^{\circ} 9\right)$ : 1360-c.

Gilgenkrantz S. Un peu de lumière sur les neurones autofluorescents: isolement du gène de la maladie de Batten. 11 (n०12) : 1752-3-nouv.

Gilgenkrantz S. Les craniosynostoses revues et corrigées par la génétique moléculaire, ou l'irrésistible ascension des récepteurs de facteurs de croissance. $11\left(n^{\circ} 12\right)$ : 1748-51-nouv.

Gilon P. Voir Henquin J.-C.

Gingras A.-C. Régulation par l'insuline de l'initiation de la synthèse protéique. 11 $\left(n^{\circ} 5\right)$ : 866-72-mns.

Ginot F. L'instrumentation automatique en biologie moléculaire. $11\left(n^{\circ} 3\right)$ : 455-64dt.

Girish S. La poly (ADP-ribose) polymérase (PARP) : à la croisée des chemins de la réparation de I'ADN et de l'apoptose cellulaire. $11\left(n^{\circ} 10\right)$ : 1487-8-nouv.

Glaichenhaus $\mathbf{N}$. Un vaccin contre la leishmaniose. 11 ( $\left.n^{\circ} 7\right)$ : 1048-9-nouv.

Glowinski J. Voir Tassin J.-P.

Gluckman E. Utilisation thérapeutique des cellules souches hématopoïétiques du sang de cordon. $11\left(n^{\circ} 1\right): 28-34$-as.

Gluckman J.-C. Le risque zéro est-il concevable en pratique médicale? 11 $\left(n^{\circ} 8\right)$ : 1160-1-lo.

Godbout R. Voir Bédard M.-A.

Godbout R. Voir Stip E.

Gogusev J. Voir Nezelof C.

Goldman M. Voir Gérard C.

Golstein P. Deux mécanismes molécu laires pour la cytotoxicité T : perforine/ granzymes et Fas. $11\left(n^{\circ} 1\right): 99-104-m n s$

Gorin I. Voir Dupin N.

Gosselin J. L'antigène CD21 est-i I'unique récepteur du virus Epstein-Barr ? $11\left(n^{\circ} 4\right)$ : 623-4-nouv

Goudeau A. Voir Poisson F.

Grandadam M. Voir Dupin N.

Grandchamp B. Voir de Verneuil H.

Grépin C. Le cœur: un programme unique de transcription et de différenciation musculaire. $11\left(n^{\circ} 3\right)$ : 395-405 -as.

Gros P. Voir Lepage P.

Grosjean H. Voir Auxillien S.

Grünfeld J.-P. Voir Chambon C.

Grünfeld J.-P. Voir Knebelmann B.

Guérin J.-F. Voir Christin-Maître S.

Guerre-Milo M. Les transporteurs d'hexoses. 11 ( $n^{\circ} 8$ ) : 1111-9-as.

Guiard B. Le rôle du complexe protéique

Hsp 70/MIM44 dans l'importation des pro- 
téines mitochondriales. $11\left(n^{\circ} 2\right): 282-3-$ nouv.

Guicheney P. Voir Carrier L.

Guilhot F. Voir Chomel J.-C.

Guillet J.-G. Voir Butor C.

H

Habert R. Voir Saez J.M.

Haddada H. Voir Fernandez N.

Hantraye P. Voir Brouillet E.

Hatem S. Voir Mercadier J.-J.

Havard S. Voir Dupin N.

Hazout A. Voir Letur-Könirsch $\mathrm{H}$

Henderson C. L'avenir thérapeutique des

facteurs neurotrophiques dans les mala

dies neuro-dégénératives. 11 ( $\left.n^{\circ} 8\right)$ : 1067 9-ed.

Henquin J.-C. Le contrôle de la sécrétion d'insuline par le glucose : signaux déclenchants et amplificateurs. 11 ( $\left.n^{\circ} 9\right): 1235$ 42-as.

Henry I. Neurofibromatose de type 1 ou maladie de von Recklinghausen : de nombreuses questions restent posées. 11 $\left(n^{\circ} 1\right)$ : 93-8-mns.

Herchuelz A. L'échange $\mathrm{Na} / \mathrm{Ca} .11\left(n^{\circ} 2\right)$ 232-8-as.

Hérold C. Implication des molécules CD dans la transmission des signaux d'activation des lymphocytes T. $11\left(n^{\circ} 5\right): 669-80$

Hervé D. Voir Tassin J.-P.

Hillaire D. Voir Richard I.

Hivroz C. Le déficit en tyrosine kinase ZAP-70 : un modèle de déficit immunitaire héréditaire pour l'analyse de l'activation et de la différenciation des lymphocytes T. 11 $\left(n^{\circ} 2\right)$ : 268-72-mns.

Hoebeke J. La seconde boucle extracellulaire des récepteurs couplés aux protéines G: une cible préférentielle pour des autoanticorps fonctionnels. $11\left(n^{\circ} 12\right): 1661$ 7-as.

Hoeben E. Voir Verhoeven G

Honoré N. Voir Zalcman G.

Huck S. Le répertoire B humain : expression normale et pathologique. $11\left(n^{\circ} 11\right)$ : 1566-75-as.

Hue L. Régulation du métabolisme hépatique par le gonflement cellulaire. 11 $\left(n^{\circ}\right.$ ) : 1243-9-as.

Hulsey T. Voir Robillard P.-Y.

Humbert J.-P. Le signal calcium dans la cellule: l'enveloppe nucléaire est-elle un réservoir à calcium ? $11\left(n^{\circ} 10\right): 1437-41-m n s$. Huraux J.-M. Voir Dupin N.

Imbert J. Voir Costello R.

Israël A. Les protéines Rel/NF- $\kappa B$ et $I \kappa B$ nouvelles données sur la structure, la fonction et la régulation. $11\left(n^{\circ} 7\right): 1017-20-m n s$.

J

Jackson C.E. Voir Richard I. Jackson C.E. Voir Duclos F.
Jacquot C. Voir Gardier A.M.

Jariel-Encontre I. Voir Carillo S

Jégou B. Tout ce que vous avez toujours voulu savoir sur le testicule sans jamais oser le demander. $11\left(n^{\circ} 4\right): 517-8$-ed. Jégou B. La cellule de Sertoli : actualisa tion du concept de cellule nourricière. 11 $\left(n^{\circ} 4\right)$ : 519-27-as.

Jordan B.R. Allemagne : enfin un pro gramme Génome humain. $11\left(n^{\circ} 8\right)$ : 1162 4-cg.

Jordan B.R. Génome humain: I'annuaire nouveau est arrivé. $11\left(n^{\circ} 12\right): 1717-19-c g$. Jordan B.R. La valse des étiquettes. 11 $\left(n^{\circ} 2\right)$ : 273-6-cg.

Josso $\mathbf{N}$. La cellule de Sertoli, une cellule endocrine. $11\left(n^{\circ} 4\right)$ : 537-46-as.

Jouannet P. Mouvement normal et pathologique du spermatozoïde humain. 11 $\left(n^{\circ} 4\right)$ : 555-62-as.

Jouannet P. Voir Spira A.

Jouvet M. Voir Mick G.

Jouvet $\mathbf{M}$. Sérotonine et sommeil : une histoire inachevée. $11\left(n^{\circ} 6\right)$ : 886-93-hd.

Jullian N. Voir Darlix J.-L.

Jurkat-Rott K. Voir Fontaine B.

Kahn A. Voir Bergeron M.

Kahn A. Voir Bursaux E.

Kahn A. Lymphopoïèse et gène E2A : le rôle spécifique d'un facteur ubiquitaire. 11 (n०3) : 482-3-nouv.

Kahn A. Voir Chambon C.

Kahn A. Voir Dreyfus J.-C.

Kahn A. Un gène inducteur de la différenciation de l'œil conservé entre les insectes et les mammifères. 11 ( $\left.n^{\circ} 5\right)$ : 776-7-nouv. Kahn A. La phosphatidyl inositol-3 kinase et la transmission des signaux : un rôle ambigu. $11\left(n^{\circ} 6\right)$ : 903-5-nouv.

Kahn A. Comment calmer les lymphocytes cytotoxiques qui veulent en faire trop? $11\left(n^{\circ} 6\right)$ : 912-3-nouv.

Kahn A. Vaccins végétaux. $11\left(n^{\circ} 6\right): 926$ nouv.

Kahn A. L'interruption du signal de l'érythropoïétine nécessite l'action d'une phosphotyrosine protéine phosphatase. 11 (n6) : 927-8-nouv.

Kahn A. Jean-Claude Dreyfus, 1916-1995. $11\left(n^{\circ} 6\right)$ : 929-32-hom.

Kahn A. Le niveau d'expression du gène de l'insuline est-il relié à la susceptibilité au diabète de type 1 ? 11 ( $\left.n^{\circ} 7\right)$ : 1042-3nouv.

Kahn A. Le récepteur du TGF $\beta$, cible des anomalies de réparation dans des formes congénitales du cancer colorectal non polyposique. $11\left(n^{\circ} 8\right)$ : 1176-7-nouv.

Kahn $A$. Le gène de l'ataxie télangiectasie code pour une protéine ressemblant à la $\mathrm{PI}-3$ kinase. 11 ( $n^{\circ} 8$ ) : 1189-90-nouv.

Kahn A. Une confirmation: le produit du gène $o b$ est bien une hormone agissant comme un lipostat. $11\left(n^{\circ} 10\right)$ : 1463-4-mns. Kahn A. Les défenses du testicule greffé: qui s'y frotte, s'y pique. $11\left(n^{\circ} 12\right): 1756$ nouv.

Kahn A. Régulation de l'élongation de la transcription par le produit du gène $V H L$ 11 ( $\left.{ }^{\circ} 11\right)$ : 1603-4-nouv.
Kahn A. Mécanismes génétiques de la maladie d'Alzheimer : un gène de susceptibilité de plus sur le chromosome 1. 11 (n० 11): 1610-1-nouv.

Kahn A. La mucoviscidose est bien due à l'altération d'un régulateur transmembranaire de la conductance. $11\left(n^{\circ} 11\right): 1612$. 13-nouv.

Kahn-Perlès B. Voir Costello R

Kaplan J. Des gènes pour les dystrophies rétiniennes des enfants. $11\left(n^{\circ} 3\right)$ : 325-35as.

Kaufmann S. Voir Girish S.

Kerleau M. Analyse coût-avantage d'une politique de prévention vaccinale de I'hépatite virale B. 11 ( $\left.n^{\circ} 10\right): 1474-7-f c$.

Ketelslegers J.-M. Voir Thissen J.-L.

Kitzis A. Voir Chomel J.-C.

Knebelmann B. En route vers la compréhension de la kystogenèse dans la polykystose rénale. $11\left(n^{\circ} 9\right): 1348-9$-nouv.

Koenig $\mathbf{M}$. Identification du gène de I'ataxie par déficit isolé en vitamine E. 11 $\left(n^{\circ} 3\right)$ : 472-3-nouv

Kokorine I. Voir Marbaix E.

Kolb J.-P. Voir Paul-Eugène/Dugas $\mathrm{N}$.

Köppler P. Voir Humbert J.-P.

Krol A. Voir Sturchler-Pierrat C.

Labie D. Un régulateur global de transcription impliqué dans l'association retard mental et $\alpha$-thalassémie (syndrome ATRX). $11\left(n^{\circ} 6\right): 910$-1-nouv.

Labie $D$. On guérit l' $\alpha$-thalassémie de la souris avec un gène humain. $11\left(n^{\circ} 11\right)$ : 1601-2-nouv.

Labie D. Où en est-on au juste avec les facteurs GATA? Redondance ou/et spécificité ? 11 (n० 11) : 1608-9-nouv.

Lacombe C. L'érythropoïétine. $11\left(n^{\circ} 7\right)$ : 947-55-as.

Lacombe D. Schizophrénie et délétions du chromosome 22q11. $11\left(n^{\circ} 12\right): 1727$. 31-mns.

Lacronique J.-F. La transmission professionnelle du SIDA. $11\left(n^{\circ} 12\right): 1725-f c$.

Lamballe F. Les récepteurs tyrosine kinases Trk: récepteurs de forte affinité des neurotrophines. 11 ( $\left.n^{\circ} 8\right)$ : 1071-80-as.

Lamour-Isnard C. Voir Deutsch J.

Lamy F. Voir Maenhaut C.

Lamy F. Voir Dupin N.

Lang D. Voir Humbert J.-P.

Lanoé J.-L. Voir Kerleau M.

Lapie P. Voir Fontaine B.

Larget-Piet D. Voir Kaplan J.

Latruffe $\mathbf{N}$. Voir Caira F.

Laurent $M$. Pour une approche dynamique du fonctionnement cellulaire. 11 $\left(n^{\circ} 1\right)$ : 119-23-hd.

Laurent M. Quand la communication scientifique empruntera les autoroutes de l'information. $11\left(n^{\circ} 10\right): 1471-3-l o$.

Lavoinne A. Voir Hue L.

Le Deist F. Voir Hivroz C.

Le Guyader H. Voir Philippe H.

Le Guyader H. Voir Deutsch J.

Le Merrer M. Voir Dreyfus J.-C.

Le Paslier D. Voir Bürglen L.

Lebrun P. Voir Herchuelz A.

Lecine P. Voir Costello R. 
Ledent C. Génétique moléculaire des affections thyroïdiennes. $11\left(n^{\circ} 2\right): 215-21$

Lefebvre S. Voir Bürglen L.

Léger J.J. Voir Eldin P.

Lehmann-Horn F. Voir Fontaine B.

Leibowitch M. Voir Dupin N.

Lejeune H. Voir Saez J.M.

Lemaire G. Voir Crépel F

Lepage $\mathbf{P}$. La glycoprotéine $P$ : de la résis tance croisée aux médicaments au transport des lipides biliaires. $11\left(n^{\circ} 3\right): 357-66$

Lepesant J.-A. Voir Deutsch J.

Leroy $\boldsymbol{X}$. Voir Deliège $D$.

Letur-Könirsch H. Pratique du don d'ovocytes : l'expérience de l'hôpital Antoine-Béclère. $11\left(n^{\circ} 4\right): 591-600-n o$. Léveillé C. Voir Aoudjit F.

Lévy J.-P. Le problème d'un vaccin contre le SIDA. 11 ( $\left.n^{\circ} 3\right)$ : 407-19-as.

Lewin M J.-M. Les inhibiteurs de la pompe à protons gastrique : mode d'action et intèrêt thérapeutique. $11\left(n^{\circ} 1\right): 62-71-a s$

Lewontin R.C. Promesses, promesses. $11\left(n^{\circ} 5\right)$ : II-V-sfg.

Libert F. Voir Parmentier M.

Lim L.E. Voir Duclos F.

Lipcey C. Voir Costello R.

Lorca T. Voir Carillo S.

Lotteau V. Présentation des antigènes qui présente quoi? $11\left(n^{\circ} 5\right)$ : 659-60-ed.

Lotteau V. Présentation des déterminants cachés. $11\left(n^{\circ} 12\right)$ : 1746-7-nouv.

Lupien S. Glucocorticoïdes et cognition. $11\left(n^{\circ} 11\right)$ : 1524-30-as.

Lustenberger P. Voir Denis M.G.

Lyonnet S. Voir Attié T.

\section{$\mathrm{M}$}

Mach B. Voir Durand B.

Macq A.-M. Voir Octave J.-N.

Maenhaut C. La cascade mitogène de I'AMPc dans la thyroïde et dans d'autres tissus. $11\left(n^{\circ} 2\right)$ : 204-13-as.

Maiter D. Voir Thissen J.-L.

Malaisse W.J. Anomalies du métabolis me insulaire dans le diabète. $11\left(n^{\circ} 2\right)$ 199-202-as.

Malviya A.N. Voir Humbert J.-P.

Mancini M. Voir Schlienger K.

Mandel J.-L. Voir Koenig M.

Manning B. St. J. Voir Strosberg A.D.

Mannoni P. Voir Chabannon C.

Marbaix E. Collagénases et métallopro téases apparentées: un rôle essentiel dans le déclenchement de la menstruation? 11 ( $n^{\circ}$ 9) : 1261-9-as.

Maréchal J. Voir Delaunay J.

Mari B. Structure et fonction des ectopeptidases du système immunitaire. $11\left(n^{\circ} 5\right)$ : 681-90-as.

Marineau C. Le gène de la neurofibromatose de type 2. $11\left(n^{\circ} 1\right): 35-42-a s$.

Martin A. Voir Raphaël M.

Martinou J.-C. La mort cellulaire programmée dans le système nerveux. 11 (n³) : 367-73-as.

Masquilier D. La voie de I'AMPc lors de la spermatogenèse : rôle clé du gène CREM. 11 ( $\left.n^{\circ} 4\right)$ : 616-20-mns.

Matter N. Voir Humbert J.-P.
Matuchansky C. Aspects familiaux du cancer recto-colique sporadique: acquis épidémiologiques récents pour le clinicien. $11\left(n^{\circ} 8\right)$ : 1158-9-mns.

Mayeux P. Voir Lacombe C

McElreavey K. Voir Barbaux S.

Meijer A.J. Voir Hue L.

Melin J. Voir Vanoverschelde J.-L.

Melki J. Voir Bürglen L.

Mercadier J.-J. Le syndrome du QT long congénital: c'est bien une affaire de canaux ioniques ! $11\left(n^{\circ} 10\right): 1453-9-m n s$.

Mérel P. Voir Marineau C.

Merten M. Mucoviscidose: vers quelle cellules pulmonaires faut-il orienter la recherche fondamentale et clinique? 11 $\left(n^{\circ} 10\right)$ : 1442-6-mns.

Méry P.-F. Voir Capiod T.

Métin C. Vers une compréhension des mécanismes de la vision aveugle. 11 (n०12) : 1767-8-nouv.

Meyer J. Voir Duclos F.

Miccoli L. Voir Oudard S.

Michel M.-L. Voir Schlienger K.

Mick G. Rythmes circadiens : leurs bases anatomiques, fonctionnelles et moléculaires. $11\left(n^{\circ} 1\right)$ : 52-61-as.

Mignon A. NO : KO. $11\left(n^{\circ} 12\right): 1743-4$ nouv.

Mignon M. Helicobacter pylori et maladie ulcéreuse : cause absolue ou cofacteur physiopathologique majeur? $11\left(n^{\circ} 1\right)$ : 113-8-hd.

Millasseau P. Voir Bürglen $L$.

Miot F. Voir Maenhaut C.

Moffett S. Voir Bouvier M

Monaco L. Voir Masquilier D.

Moomaw C. Voir Duclos F.

Morange $\mathbf{M}$. De la découverte de l'ADN au drame du sang contaminé : ce que savoir veut dire. 11 ( $\left.n^{\circ} 8\right)$ : 1167-8-hms.

Moreau-Gaudry F. Voir de Verneuil H.

Mornet D. Voir Eldin P

Mossalayi D.M. Voir Paul-Eugène/Dugas N.

Mota Vieira L. Une autre maladie neurodégénérative due à l'expansion du triplet CAG : la maladie de Machado-Joseph. 11 $\left(n^{\circ}\right.$ 1) : 109-11-mns.

Mouillac B. Voir Bouvier M

Moulard M. Rôle de la maturation protéolytique des glycoprotéines d'enveloppe virale. $11\left(n^{\circ} 1\right)$ : 73-80-as.

Mourad W. Voir Aoudjit F.

Munnich A. Voir Bürglen L.

Munnich A. Voir Kaplan J.

Munnich A. Voir Dreyfus J.-C.

Nalpas B. Voir Kerleau M

Nantel F Voir Bouvier M.

Nemer M. Voir Durocher D.

Nemer M. Voir Grépin C.

Nezelof C. Les réarrangements du gène NPM (nucléophosmine) en $5 q 35$. Un élément nouveau dans la compréhension et le démembrement des lymphomes anaplasiques à grandes cellules, CD30+. 11 (n०5) : 778-9-nouv.

Nicolas J.-F. Les pemphigus, maladies auto-immunes acquises de l'adhérence des kératinocytes. 11 ( $\left.n^{\circ} 7\right):$ 995-1003-as.
Nicot T. Voir Denis F.

Nordmann Y. Voir de Verneuil H.

Nurden A.T. Voir Caen J.-P.

Octave J.-N. Le précurseur du peptide amyloïde dans la maladie d'Alzheimer. 11 ( $\left.n^{\circ} 9\right)$ : 1251-9-as.

Olive D. Voir Costello R.

Olivennes F. Voir Letur-Könirsch H.

Olofsson B. Voir Zalcman G.

Olschwang S. Voir Richard S

Onteniente B. Mieux comprendre le rôle des neurotrophines dans le système nerveux grâce à l'invalidation génique. 11 $\left(n^{\circ} 8\right): 1141-3-m n s$

Opperdoes F.R. Conception rationnelle de nouveaux médicaments contre la maladie du sommeil. 11 ( $\left.n^{\circ} 9\right)$ : 1305-13-as

Ottmann M. Voir Darlix J.-L.

Ouaaz F. Voir Paul-Eugène/Dugas N.

Ouachi K. Voir Koening M.

Oudard S. Hexokinase mitochondriale, enzyme clé de la bioénergétique cellulaire: une cible potentielle pour une thérapeutique anticancéreuse. $1\left(n^{\circ} 8\right): 1121-9$-as.

Pacaud P. Voir Capiod T.

Palfi S. Voir Brouillet E.

Panisset M. Voir Bédard M.-A.

Panneels V. Voir Boeynaems J.-M.

Papiernik E. Voir Sornette D.

Pariat M. Voir Carillo S.

Parma J. Voir Ledent $C$.

Parmentier M. La famille des récepteurs couplés aux protéines $\mathrm{G}$ et ses orphelins.

$11\left(n^{\circ} 2\right)$ : 222-31-as.

Passos-Bueno M.-R. Voir Richard I.

Pasturaud P. Voir Richard I.

Paul-Eugène/Dugas $\mathbf{N}$. Rôle du CD23 dans la production de monoxyde d'azote par les monocytes humains. 11 ( $\left.n^{\circ} 12\right)$ : 1653-9-as.

Paulin D. Voir Colucci-Guyon E.

Pays E. Antigènes variables et non variables des trypanosomes africains. 11 $\left(n^{\circ} 2\right)$ : 261-7-as.

Pelletier R.M. Le rôle des jonctions intercellulaires dans le fonctionnement de la barrière hémato-testiculaire. $11\left(n^{\circ} 4\right)$ : 605-9-mns.

Peschanski M. La Society for Neuroscience, comme un ouragan ! $11\left(n^{\circ} 2\right)$. 277-fc

Peschanski M. Tyrosine hydroxylase : trois vecteurs viraux pour un gène. 11 $\left(n^{\circ} 3\right)$ : 474-5-nouv.

Peschanski $M$. Le GDNF, après ou avant tous les autres? $11\left(n^{\circ} 3\right)$ : 480-1-nouv.

Peschanski M. Les progéniteurs du système nerveux immortalisés, vecteurs de thérapie génique. $11\left(n^{\circ} 6\right): 906-8$-nouv. Peschanski $M$. Une nouvelle vision de l'amertume. 11 ( $n^{\circ}$ 9) : 1339-40-nouv.

Peschanski M. Premiers pas dans la neurobiologie du cerveau qui pense. $11\left(n^{\circ} 9\right)$ : 1350-1-nouv.

Petit C. Une myosine non conventionnel- 
le à l'origine de l'une des formes génétiques du syndrome de Usher. 11 ( $\left.n^{\circ} 8\right)$. 1181-2-nouv.

Petit C. Une nouvelle arylsulfatase à l'origine de la chondrodysplasie ponctuée ré cessive liée au chromosome $X$ et peut-être d'une embryopathie médicamenteuse. 11 $\left(n^{\circ} 10\right)$ : 1492-4-nouv.

Peyron E. Voir Nicolas J.-F.

Phelouzat M.-A. Apoptose et sénescence. $11\left(n^{\circ} 6\right)$ : 894-900-hd.

Philippe B. Voir Octave J.-N.

Philippe H. Que savons-nous de I'histoire évolutive des eucaryotes? 1. L'arbre universel du vivant et les difficultés de la re construction phylogénétique. $11\left(n^{\circ} 8\right):$ I XV-sfg.

Picard B. Voir Denamur E

Picciotto $M$. Sous-unité $\beta 2$ du récepteur nicotinique et apprentissage. $11\left(n^{\circ} 6\right)$ : 922-4-nouv.

Piechaczyk M. Voir Carillo S

Pineau C. Voir Jégou B.

Pinell P. Naissance et développement de la radiothérapie en France. $11\left(n^{\circ} 11\right)$ : 1596-99-hms.

Pinet V. HLA-DM ou comment rendre les molécules HLA de classe II présentables. $11\left(n^{\circ} 5\right)$ : 747-51-mns.

Pirson I. Voir Maenhaut C.

Plassart E. Voir Fontaine B.

Poirier G.G. Voir Girish S

Poisson F. Le virus de I'hépatite delta: un mode de réplication bien singulier. 11 $\left(n^{\circ} 10\right)$ : 1379-87-as.

Poupon A. Voir Oudard S.

Poupon M.-F. Voir Vandenbunder B.

Poupon M.-F. Voir Oudard S.

Privat A. Voir Ridet J.-L.

Proust J. Voir Phelouzat M.-A.

Puech F. Voir Cadore B.

Quadri R.A. Voir Phelouzat M.-A.

Raoul-Duval A. Voir Letur-Könirsch H Raphaël M. Les lymphomes du SIDA. 11 (n०5) : 713-22-as.

Rappaport R. Professeur Pierre Royer 1917-1995. 11 (n $\left.{ }^{\circ} 5\right)$ : 791-2-hom.

Reboul J. Voir Fontaine B.

Reboullet S. Voir Bürglen $L$.

Renauld J.-C. L'IL9 et son récepteur, un modèle pour l'oncogenèse des lymphocytes T. 11 ( $\left.n^{\circ} 9\right)$ : 1289-94-as.

Renneville $M$. Les théories biologiques de la criminalité. $11\left(n^{\circ} 12\right): 1720-4-h m s$

Resche F. Voir Richard S.

Rey R. Voir Josso N.

Richard I. Une protéase, la calpaïne 3, est responsable d'une forme de dystrophie des ceintures. $11\left(n^{\circ} 4\right)$ : 637-40-nouv.

Richard I. Voir Duclos F.

Richard S. La maladie de von Hippel-Lindau $11\left(n^{\circ} 1\right): 43-51$-as.

Richard Y. Signaux de la coopération T-B et production d'anticorps. $11\left(n^{\circ} 5\right) \cdot 691$ 702-as.
Ridet J.-L. Données anatomiques sur l'organisation des systèmes à mono-amines un exemple de communication interneuronale sans synapse ? $11\left(n^{\circ} 6\right): 861-5-m n s$. Rime C.-S. Voir Fontaine B.

Robillard P.-Y. Nouvelles perspectives de prévention de l'hypertension gravidique. L'approche immunologique. 11 $\left(n^{\circ} 1\right)$ : 130-1-nouv.

Roger P. Voir Maenhaut C.

Roingeard $\mathbf{P}$. Voir Poisson $F$.

Roques B. Voir Darlix J.-L.

Rossier B. Identification d'un gène de l'hypertension artérielle. $11\left(n^{\circ} 2\right): 296-7$ nouv.

Roucoux A. Le contrôle nerveux de l'orientation du regard. 11 ( $\left.n^{\circ} 9\right)$ : 1323-31-as.

Roudaut C. Voir Richard I.

Rouleau G.A. Voir Marineau C.

Rousseau F. Voir Dreyfus J.-C.

Roy R. TFIIH (BTF2), à l'interface de trois processus cellulaires : transcription, reparation et cycle cellulaire. $11\left(n^{\circ} 6\right): 879-82$ mns.

Rozet J.-M. Voir Kaplan J.

Russo-Marie F. Voir Émilie D.

Ruysschaert J.-M. Rôle des motifs protéiques amphiphiles dans les processus de reconnaissance. $11\left(n^{\circ} 2\right)$ : 247-52-as.

Saez J.M. Le contrôle des fonctions différenciées de la cellule de Leydig. $11\left(n^{\circ} 4\right)$. 547-53-as.

Sage J. Souris transgéniques et lignées cellulaires : outils pour une approche moléculaire de la différenciation germinale $11\left(n^{\circ} 4\right): 611-5-m n s$

Sansonetti P. Voir Zychlinsky A. Sassone-Corsi P. Voir Masquilier D. Schiffmann S.N. Implication de l'adénosine dans les noyaux de la base : interactions avec le système dopaminergique. 11 $\left(n^{\circ} 2\right)$ : 169-76-as.

Schlienger K. Vaccination contre le SIDA: évaluation chez les primates. 11 $\left(n^{\circ} 7\right)$ : 985-93-as

Schlotter F. Voir Masquilier D.

Schneider-Maunoury S. Voir Topiko P Schwartz J.-C. La neuropharmacologie au présent et au futur. 11 ( $\left.n^{\circ} 3\right)$ : 375-81-as Schwartz K. Voir Carrier L.

Schwartz K. Voir Bonne G.

Sellal F. L'action amnésiante des benzodiazépines chez l'homme. $11\left(n^{\circ} 11\right)$. 1519-23-as.

Selva J. Voir Letur-Könirsch $\mathrm{H}$.

Serres C. Voir Jouannet $P$.

Sicard D. Plainte pour assistance à personne en danger. $11\left(n^{\circ} 1\right): 148-c$.

Slaughter C. Voir Duclos F.

Sornette D. Modèle mathématique de la parturition humaine. $11\left(n^{\circ} 8\right): 1150-3$ mns.

Soü̈ed E. Voir Kaplan J.

Soulier J. Herpesvirus associé à la mala die de Kaposi KSHV/HHV8 : nouveaux résultats et nouvelles questions. $11\left(n^{\circ} 11\right)$ 1605-7-nouv

Soussi T. Hyperméthylation, cancer et inactivation des gènes suppresseurs de tu- meur $p 16^{\text {CDKN2MTS1 }}$ et HIC-1. 11 ( $\left.n^{\circ} 9\right): 1346$ 7-nouv.

Spira A. La qualité du sperme a-t-elle baissé au cours des cinquante dernières années ? $11\left(n^{\circ} 4\right): 621-2-f c$.

St-Arnaud R. Expression anormale du proto-oncogène $c$-fos dans l'os atteint de dysplasie fibreuse. $11\left(n^{\circ} 9\right)$ : 1342-3-nouv. Steff A.-M. Voir Carillo S.

Stip E. Cortex préfrontal, monoamines et neuropsychologie de la schizophrénie. 11 $\left(n^{\circ} 11\right): 1531-9$-as

Strosberg A.D. Le récepteur $\beta 3$-adrénergique: un gène de poids. $11\left(n^{\circ} 10\right): 1460$ 2-mns.

Sturchler-Pierrat C. Sélénium, sélénoprotéines: une autre lecture du code génétique. $11\left(n^{\circ} 8\right)$ : 1081-8-as.

Sunada Y. Voir Duclos F.

Szepetowski P. Voir Courseaux A

Takeda K. Voir Capiod T.

Talbot P. Implication des virus dans la sclérose en plaques. $11\left(n^{\circ} 6\right): 837-43$-as.

Tanzer J. Voir Chomel J.-C.

Tassin J.-P. L'hétéro-régulation des récepteurs ou la présence d'une relation fonctionnelle entre deux ensembles neuronaux. 11 ( $\left.n^{\circ} 6\right)$ : 829-36-as.

Tavitian A. Voir Zalcman G.

Testart J. La procréation : une fonction naturellement inhibée. $11\left(n^{\circ} 3\right)$ : 447-53-

Thieffry D. Voir Thomas R.

Thiemermann C. Inhibition des NO synthases dans la défaillance circulatoire: effet bénéfique ou délétère? $11\left(n^{\circ}\right.$ 12) : 1643-51-as.

Thissen J.-P. Nutrition et axe somatotrope : des mécanismes moléculaires à la clinique. $11\left(n^{\circ} 9\right)$ : 1225-33-as.

Thivolet J. Voir Nicolas J.-F.

Thomas G. Voir Marineau C.

Thomas G. Dix ans de recherche sur les prédispositions génétiques au développement de tumeurs. $11\left(n^{\circ} 3\right): 336-48$-as.

Thomas $R$. Les boucles de rétroaction, rouages des réseaux de régulation biologiques. $11\left(n^{\circ} 2\right)$ : 189-97-as.

Thonneau P. Voir Spira A

Tiollais P. Voir Schlienger K. Tischfield J.A. Voir Richard I.

Tolédo F. Voir Debatisse M.

Tomé F. M.S. Voir Duclos F

Tomé F. M.S. Voir Fardeau M.

Topiko $\mathbf{P}$. Le contrôle de la myélinisation du système nerveux périphérique, un rôle inattendu pour le facteur de transcription Krox-20. $11\left(n^{\circ} 1\right)$ : 142-3-nouv.

Toussaint C. Les hélicases. $11\left(n^{\circ} 10\right)$ : 1389-98-as.

Trovero F. Voir Tassin J.-P

Underwood L.E. Voir Thissen J.-L. 


\section{V}

Vainchenker $\boldsymbol{W}$. Voir Coulombel L. Vainchenker $\boldsymbol{W}$. Voir Wendling $F$

Valé-Santos J. Voir Fontaine B.

Valiquette M. Voir Bouvier M.

Van den Eynde B. Voir Boon T.

Van Eylen F. Voir Herchuelz A

Van Sande J. Voir Ledent C.

Van Sande J. Voir Boeynaems J.-M.

Vandenbunder B. Découverte d'un nouve inhibiteur de l'angiogenèse, l'angiostatine. Implications pour la biologie et la thérapie des métastases. $11\left(n^{\circ} 2\right)$ : 284-6-nouv.

Vandenbunder B. Les micrométastases adoptent-elles la stratégie de Pénélope ? 11 (n०5): 772-3-nouv.

Vandenbunder B. Repenser les oncogènes en 1995? $11\left(n^{\circ} 10\right)$ : 1465-70-hd.

Vanderhaeghen J.-J. Voir Schiffmann S. N.

Vanherweghem J.-L. Voir Dumont J.E.

Vanoverschelde J.-L. Physiopathologie de l'hibernation myocardique chronique apports de la tomographie par émission de positons, $11\left(n^{\circ} 9\right)$ : 1315-22-as.

Vanweyenberg V. Voir Erneux C.

Varet B. L'érythopoïétine : quel avenir ?

$11\left(n^{\circ} 7\right)$ : 943-5-ed.
Vassart G. Voir Ledent C.

Vassart G. Voir Parmentier M.

Vasseur-Cognet $\mathbf{M}$. La combinaison des deux inducteurs $\mathrm{C} / \mathrm{EBP} \alpha$ et RXR/PPAR 2 détermine la différenciation des fibroblastes en adipocytes. $11\left(n^{\circ} 4\right)$ : 625-6-nouv.

Vauge C. Voir Sornette D.

Veithen A. Voir Cupers P.

Velu T. Voir Gérard C.

Venet A. Voir Butor C

Verhoeven G. Les cellules péritubulaires. $11\left(n^{\circ} 4\right)$ : 601-4-mns.

Veuille M. Richard C. Lewontin. $11\left(n^{\circ} 5\right)$ l-sfg.

Vezina P. Voir Tassin J.-P.

Vilain E. Voir Barbaux S.

Villiers C. C3, protéine du complément: une molécule aux multiples capacités. 11 $\left(n^{\circ} 10\right):$ 1419-29-as.

Viollet L. Voir Bürglen L.

Vitale N. Protéines $\mathrm{G}$ trimériques et transport vésiculaire : implication d'une protéine Go granulaire dans une étape de l'exocytose contrôlée. $11\left(n^{\circ} 7\right)$ : 1034-6-mns.

Viville S. Recombinaison homologue: nouveaux vecteurs, nouvelles perspectives. $11\left(n^{\circ} 5\right): 735-46$-as.
W

Weissenbach J. Voir Bürglen L.

Weissenbach $\mathbf{J}$. Le génome humain entre médecine et science. $11\left(n^{\circ} 3\right): 317-23-a s$. Wendling F. Rôle inattendu du facteur de transcription NF-E2 dans la maturation terminale du mégacaryocyte et la production des plaquettes. $11\left(n^{\circ} 10\right)$ : 1503-4-nouv. Wijns W. Voir Vanoverschelde J.-L.

Wilmotte R. Voir Delaunay J.

\section{$\checkmark$}

Yu C. Voir Darlix J.-L.

Z

Zalcman G. Participation de la cascade des protéines Rho à la régulation du cytosquelette : rôle possible dans les mécanismes d'oncogenèse. $11\left(n^{\circ} 11\right)$ : 1551-6-as.

Zatz M. Voir Richard I.

Zeviani M. Voir Bürglen L.

Zouali M. Voir Huck S.

Zychlinsky A. Apoptose, interleukine-1 et shigellose. $11\left(n^{\circ} 1\right)$ : 128-9-nouv. 